\title{
Evaluation of a computer-assisted method for individualized anticoagulation: Retrospective and prospective studies with a pharmacodynamic model
}

\begin{abstract}
We studied the effectiveness of a computer program based on a mathematic model of warfarin dynamics in assisting with the initial phases of anticoagulation. In retrospective evaluations the program was successful in predicting prothrombin complex activity $(P C A)$ responses for three different groups of subjects, indicating that the model is a good representation of the physiologic system. In a prospective evaluation of the program a computer-assisted group of 10 patients was compared with a control group of 10 patients who did not receive computer assistance. Because of the program's very conservative upper limits for warfarin dosage in the first few days of therapy, the computer-assisted patients required slightly more time (6 days), on the average, to first reach PCA values in the $20 \%$ to $30 \%$ therapeutic range than did the control patients (4.8 days). After the desired PCA had been achieved, however, the computer-assisted group remained within the therapeutic range for $83 \%$ of the time compared to only $60 \%$ for the control group. This difference was due primarily to much less overanticoagulation of the computer-assisted patients than of the controls.
\end{abstract}

Peter H. Abbrecht, M.D., Ph.D., Timothy J. O'Leary, M.D., Ph.D., and

Douglas M. Behrendt, M.D. Bethesda, Md., and Ann Arbor, Mich.

Departments of Physiology and Internal Medicine, Uniformed Services University of the Health Sciences, and Laboratory of Pathology, National Cancer Institute, Bethesda, and Section of Thoracic Surgery, Department of Surgery, University of Michigan Medical School, Ann Arbor

Our objective was to evaluate a new computer-assisted method for individualizing anticoagulant therapy. Initial anticoagulation with oral drugs is a difficult task for the physician. Patients vary widely in their responses to oral anticoagulants. Although attempts have been

\footnotetext{
This research was supported in part by grant NSF-ENG-77-0779 from the National Science Foundation, and by a grant from the Michigan Heart Association.

Received for publication Aug. 14, 1981.

Accepted for publication Feb. 20, 1982.

Reprint requests to: Dr. Peter H. Abbrecht, Department of Physiology, Uniformed Services University of the Health Sciences, 4301 Jones Bridge Rd., Bethesda, MD 20814.
}

made to predict individual anticoagulant dosage on the basis of characteristics such as body weight, ${ }^{5}$ no simple laboratory test or physical measurement has been found that can be used to estimate reliably a patient's maintenance dose before beginning therapy. In addition, the maximal effect of a single dose of warfarin on the prothrombin complex activity (PCA) develops 36 to $54 \mathrm{hr}$ after the dose is given, so that the effects of daily dosing are cumulative and difficult to predict on an intuitive basis.

Methods for initiating oral anticoagulation should minimize the time required to achieve 
steady-state PCA values within the therapeutic range while protecting the patient from the possibility of overdosage and hemorrhage. It is difficult to evaluate the degree to which conventional methods achieve this goal, since no detailed studies have been reported on variations in the PCA during the initiation of oral anticoagulation. There are, however, several studies that document the fact that PCA control tends to be suboptimal even during the maintenance phase. Brotman ${ }^{2}$ found that under most circumstances one could expect to have a patient within the therapeutic range no more than $55 \%$ to $65 \%$ of the time and Mosely et al. ${ }^{6}$ reported that at any given time only $65 \%$ of their patients were within therapeutic limits. Shapiro et al. ${ }^{15}$ found that patients treated with oral drug were within the therapeutic range $71 \%$ of the time, but the therapeutic limits for PCA they used (11\% to $26 \%$ ) were much wider than is usually accepted at present. Pollard et al. ${ }^{10}$ reported results similar to those of Shapiro et al. The large variations in PCA that occur during long-term anticoagulation underscore the difficulties of dosage determination during the initiation of anticoagulation when there is no previous experience from which to predict a patient's response. As a result, the initial phase of anticoagulation is often unsatisfactory.

Despite the difficulties it is very important to identify an appropriate maintenance dose as soon as possible after beginning therapy. Inadequate anticoagulation may increase the risk of thrombosis, while overanticoagulation carries the risk of severe hemorrhage. Because of the importance and difficulty of achieving adequate anticoagulation rapidly and safely, an attempt to use a more rigorous method for estimating anticoagulant dosage during initiation phases is justified.

Several groups have recently reported methods that correlate either the time required to reach a specified level of anticoagulation during the loading phase ${ }^{12,13}$ or the area under the prothrombin time-dose curve ${ }^{19}$ with the ultimately required maintenance dose. While these methods are an important advance, they have not proved to be suitable for all patients. ${ }^{4,5}$

In another approach, computer models have been used to predict PCA response to warfarin during retrospective study of the initial phase of anticoagulation ${ }^{16,}{ }^{17}$ and prospectively during long-term anticoagulation, ${ }^{3,}{ }^{18}$ but they have not been used as an adjunct during the initiation of oral anticoagulant therapy. Mathematic models suitable for assisting in initial anticoagulation have been described, ${ }^{7}$ but none has been used in a computer program to assist the physician. None of the reported models provides a mechanistic framework potentially useful for the study of other aspects of warfarin pharmacology, such as interactions of warfarin with other drugs.

We have approached the problem by proposing a model ${ }^{8,9,11}$ of warfarin effect on plasma prothrombin activity that is based in part on the enzyme-substrate formulation of Sellers and Koch-Wesser. ${ }^{14}$ Our model assumes rapid absorption of warfarin so that the total body warfarin at a time $t$ is given by

$$
Q(t)=\sum_{i=1}^{n} C_{i} \exp \left[\left(t-t_{i}\right)\right]
$$

where $\mathrm{Q}(\mathrm{t})$ is the total body warfarin $(\mathrm{mg}), \mathrm{k}_{\mathrm{e}}$ is the warfarin elimination rate constant (days ${ }^{-1}$ ), and $C_{i}$ is the dose of warfarin (in $\mathrm{mg}$ ) given at time $t_{1}$.

Free warfarin depresses the prothrombin complex activity in a time-dependent manner according to the differential equation

$$
\frac{\mathrm{dP}}{\mathrm{dt}}=\mathrm{S}_{\mathrm{m}}\left(1-\frac{\alpha \mathrm{Q}(\mathrm{t})}{\alpha \mathrm{Q}(\mathrm{t})+\mathrm{k}_{\mathrm{m}}}\right)-\mathrm{k}_{\mathrm{p}} \mathbf{P}
$$

where $\alpha$ is the free fraction of warfarin divided by the distribution volume $\left(l^{-1}\right), S_{\mathrm{m}}$ is the maximum rate of prothrombin complex synthesis (\% of control/day), $\mathrm{k}_{\mathrm{p}}$ is the prothrombin complex degradation rate constant (days ${ }^{-1}$ ), $\mathrm{P}$ is the prothrombin complex activity in plasma ( $\%$ of control), and $\mathrm{k}_{\mathrm{m}}$ is the free plasma warfarin concentration at half maximal $\mathrm{P}(\mathrm{mg} / \mathrm{l})$.

The prothrombin time, measured by the Quick technique, is converted to percent activity by the relationship $\mathrm{PCA}=\left[(\text { Protime-B) } / \mathrm{A}]^{-1}\right.$ (where, for example, $A=230$ and $B=8.2$ for group $\mathrm{R}-1$, defined below).

Here we summarize the success of a computer program based on the above model in predicting prothrombin complex response to warfarin in retrospective studies of normal volunteers and 
hospitalized patients. We also present the results of a trial of the model's effectiveness in guiding therapy in a prospective study in patients receiving anticoagulant therapy after cardiac surgery and pulmonary embolism.

\section{Brief description of the program}

In general, the parameters $\alpha, \mathrm{k}_{\mathrm{e}}, \mathrm{k}_{\mathrm{m}}, \mathrm{k}_{\mathrm{p}}$, and $S_{m}$ in the system equations must be determined for each patient. However, since the free fraction of warfarin would be expected to be relatively constant in patients not receiving drugs that affect warfarin binding, ${ }^{14}$ the same value for $a:(0.01)^{14}$ was used for all of the subjects in the studies presented here. This reduced the number of parameters that needed to be identified from the experimental data and thus enabled earlier identification of the other parameters. However, the program could be altered to allow for situations in which the fraction of unbound warfarin differs from normal, e.g., when the patient is also receiving drugs such as phenylbutazone that decrease warfarin binding. In cases in which there is no competitive drug binding, a different $\alpha$ would change the value of $\mathrm{k}_{\mathrm{m}}$ computed for any individual patient proportionately and would not affect his or her recommended doses or predicted PCAs. The parameters $\mathrm{k}_{\mathrm{e}}$ and $\mathrm{k}_{\mathrm{m}}$ are determined for each patient by a nonlinear least-squares procedure that minimizes the sums of the squares of the differences between measured PCA values and those predicted by the model for the corresponding times. There are a variety of identification techniques that could be used to obtain the best combination of values for the parameters $\mathrm{k}_{\mathrm{e}}$ and $\mathrm{k}_{\mathrm{m}}$. To minimize computation time, however, a set of possible parameter values that includes 10 different $k_{\mathrm{e}}$ values and 10 different $\mathrm{k}_{\mathrm{m}}$ values is examined to determine which combination of the two parameters gives the lowest sum of squares of the differences between measured and predicted values. ${ }^{9}$

In operation the program requires as initial information the value of the PCA at the time that anticoagulation is begun. Subsequently, the daily doses of warfarin and PCA values are entered as obtained. Since early in the course of anticoagulation there are insufficient data for more meaningful recommendations, doses of 5
Table I. Program limitations on maximum warfarin dosage recommendations

\begin{tabular}{c|c}
\hline $\begin{array}{c}\text { Highest dose given } \\
\text { previously (mg/day) }\end{array}$ & $\begin{array}{c}\text { New maximum dose } \\
(\text { mg/day })\end{array}$ \\
\hline 5.0 & 7.5 \\
7.5 & 10.0 \\
10.0 & 15.0 \\
15.0 & 20.0 \\
\hline
\end{tabular}

$\mathrm{mg}$ are given on each of the first 2 days of therapy, regardless of PCA values. Thereafter, after identification of the patient parameters, the program uses the system equations to predict the daily maintenance dose that will maintain the PCA as close to $25 \%$ (the middle of our therapeutic range) as possible. This is offered as the next suggested dose, as long as it does not fall outside of certain limits intended to keep the recommended dose from rising too rapidly early in therapy and it does not exceed $20 \mathrm{mg} /$ day. The maximum dose recommended by the program is set as follows: day $3,7.5 \mathrm{mg}$; day 4,10 $\mathrm{mg}$; day $5,15 \mathrm{mg}$; and thereafter, $20 \mathrm{mg}$. Additional constraints are placed on the maximum allowable increase in dose according to the schedule in Table I. By placing these constraints on the program, we sought to prevent excessively large doses being recommended during the early phases of anticoagulation, when the patient parameters have not yet been identified reliably, even though this limitation may slightly increase the time required initially to decrease the PCA measurement to the therapeutic range.

The program provides a set of instructions that may be displayed on a terminal or printer. After reading the instructions, which requires only 2 or $3 \mathrm{~min}$, the physician may use the program in an interactive fashion in which the computer prompts the user for all required input. When he first wishes assistance in anticoagulating a patient, the physician creates a file to hold all the patient's anticoagulation data. Any time after this he uses the dosing program, which asks for the most recent warfarin dosages and PCA values. The program then computes the dynamic parameters, the expected responses to several possible doses of warfarin, and the recommended dose. These values are then dis- 


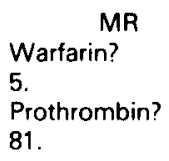

Most Probable True Protime 30 .

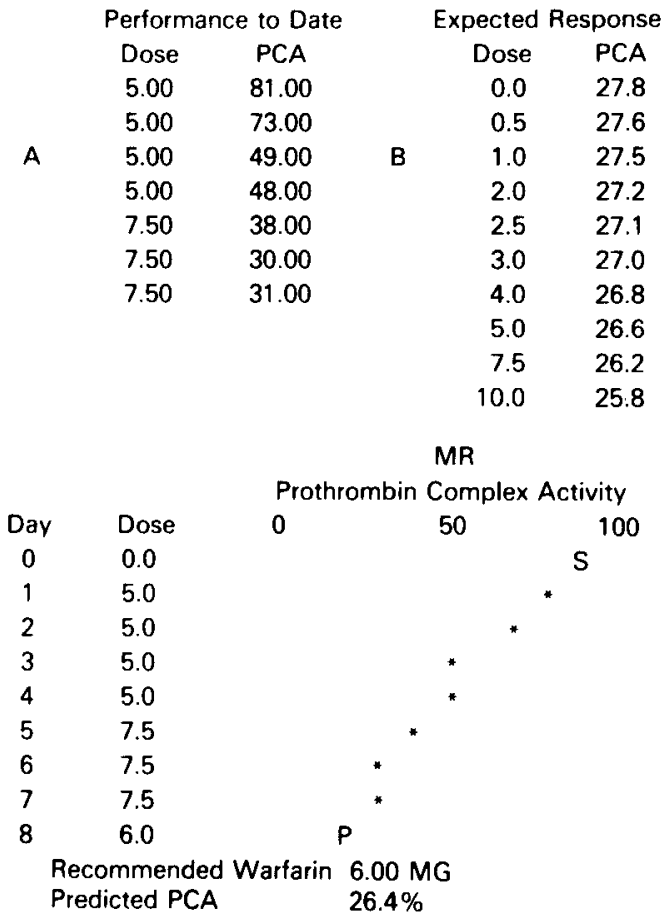

Fig. 1. Example of computer terminal output for a patient who received six daily warfarin doses. "Most probable PCA" is derived from an average of the most recent measurement and the most recent value computed by the program. Table A summarizes the dosage history and PCA response to date. Table B shows values for the next day's PCA predicted by the program for different warfarin doses.

played at the terminal along with graphic and tabular summaries of the patient's anticoagulation therapy to date. Although the program works best if daily PCA measurements are available, it can be used even for patients for whom some data are missing. The output from a typical terminal session is shown in Fig. 1.

\section{Retrospective evaluation of program performance}

Retrospective evaluations of the program were done to assess its reliability and safety before it was used for prospective guidance of patient therapy. To obtain as broad as possible an evaluation, we used data from our own laboratories and that reported by another laboratory. ${ }^{1}$ In the retrospective studies the program was used to predict each patient's dynamic parameters for each day by use of all of the previously measured PCA values for that patient. From these parameters and the prescribed dose the program then predicted the patient's PCA value for the next day (henceforth referred to as the "predicted" PCA response). Beginning at the initiation of therapy, the calculation was repeated for each subsequent day of treatment.

Three different groups of subjects were used in the retrospective studies. Group R-1 consisted of 10 normal men at the University of Michigan Medical Center (UMMC) who had received $7.5 \mathrm{mg} /$ day sodium warfarin for 14 days. Group R-2, data for which were taken from a study reported by Aggeler and O'Reilly, ${ }^{1}$ consisted of 15 normal subjects given 10 $\mathrm{mg} /$ day warfarin for 6 days. Group R-3 consisted of 17 UMMC patients selected randomly from the records of patients anticoagulated after cardiac surgery or pulmonary embolism.

The program's predictions of warfarin's effects on PCA were in all cases reasonable; the accuracy of these predictions tended to increase as more data became available for each patient. In most cases the predicted PCA values were quite close to the measured values by the third to fourth day after the beginning of treatment; this was in accordance with the results of our previous investigations. ${ }^{9}$ The maintenance dose recommended by the program usually became stable (defined as remaining within a $1.0-\mathrm{mg}$ range for 3 successive days) by the fourth to sixth day after starting warfarin administration (Table II). The program's dosage recommendations appeared reasonable in all cases.

To obtain a quantitative estimate of program performance, we calculated the normalized deviation of the predicted PCA value from the measured PCA value

$\mathrm{E}=\mid$ Observed PCA -

Predicted PCA | /Observed PCA

for each data point. Fig. 2 compares the average daily PCA deviations of groups R-2 and R-3. The average deviations decreased with time for 
Table II. Cumulative percentage of patients whose computer-recommended doses had stabilized by given day of therapy

\begin{tabular}{c|c|c|c}
\hline Day & $\begin{array}{c}\text { Retrospective } \\
\text { (group R-3) }\end{array}$ & $\begin{array}{c}\text { Computer- } \\
\text { assisted } \\
\text { (group P-1) }\end{array}$ & $\begin{array}{c}\text { Control } \\
\text { (group P-C) }\end{array}$ \\
\hline 1 & 17 & & \\
2 & 17 & & 22 \\
3 & 33 & 10 & 67 \\
4 & 59 & 40 & 78 \\
5 & 67 & 70 & 89 \\
6 & 83 & 80 & 89 \\
7 & 91 & 90 & 89 \\
\hline
\end{tabular}

both groups due to increasingly better estimates of the dynamic parameters as more data points became available. The smaller average deviations found for group R-2 compared to group $\mathrm{R}-3$ were probably due to the constant dosing schedule used by Aggeler and O'Reilly. ${ }^{1}$ It is also likely that the patients in group R-3 were exposed to more factors that affect warfarin response, such as the presence of interacting drugs, than were the normal subjects of group R-2.

The generally good agreement between the predicted and observed PCA values in all three retrospective trials established the model as a good representation of the physiologic system with adequate reliability to justify a prospective trial in patients.

\section{Prospective evaluation of program performance}

Program assistance was provided for a total of 10 patients undergoing initial anticoagulation. All but one patient considered in this phase of the study were receiving anticoagulant therapy postoperatively on the cardiac surgery service at UMMC. These patients are routinely placed on prolonged therapy after coronary artery bypass surgery or implantation of prosthetic heart valves. The remaining patient was anticoagulated after acute pulmonary embolism. The house staff responsible for the patients' clinical care decided when oral anticoagulant should be initiated.

Patients were considered eligible for our study if: (1) they had PCA of $70 \%$ or more at the onset of oral anticoagulation, (2) they were

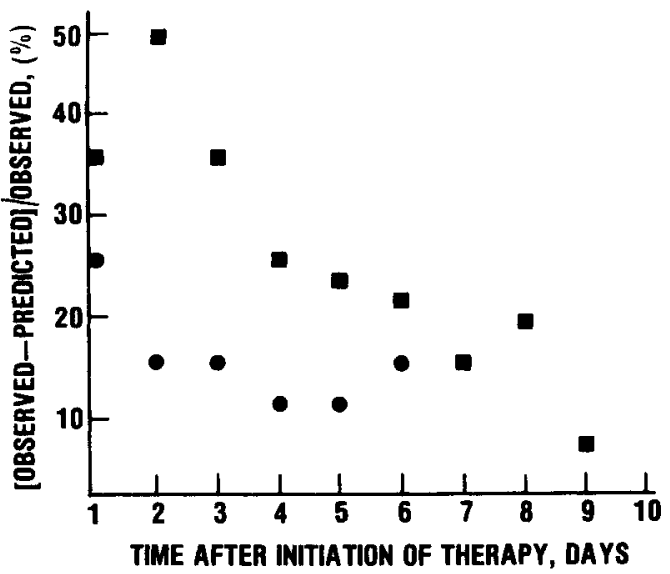

Fig. 2. Normalized average deviations of predicted PCA values from observed values in two of the retrospective studies. Points shown as (๑) were computed from the data of Aggeler and O'Reilly' (group R-2). Points shown as (a) were computed for UMMC patients (group R-3).

afebrile, and (3) they had received either no warfarin or not more than two 5-mg doses at the time we were informed of their availability for study.

A control group (group P-C) of 10 patients was anticoagulated without computer assistance. These patients were matched with the computer-assisted patients on the basis of age and diagnostic category and were treated on the same services as the computer-assisted group. Thus, the control group also consisted of nine patients who had undergone heart surgery and one patient anticoagulated for thrombophlebitis. Mean age for each group was $52 \mathrm{yr}$.

Each day, shortly after PCA values were received from the anticoagulation laboratory, we placed sheets in the order books informing the house staff of the PCA for that day and the program's current dosage recommendations. The house staff either prescribed the recommended dosage or modified it on the basis of clinical judgement.

Fig. 3 shows the recommended warfarin doses for one of these patients along with the computer-predicted and observed PCA values. The responses of the remaining patients were on the same order. Fig. 4 gives the average percentage deviations of the predicted PCA values from the observed values for the computer- 


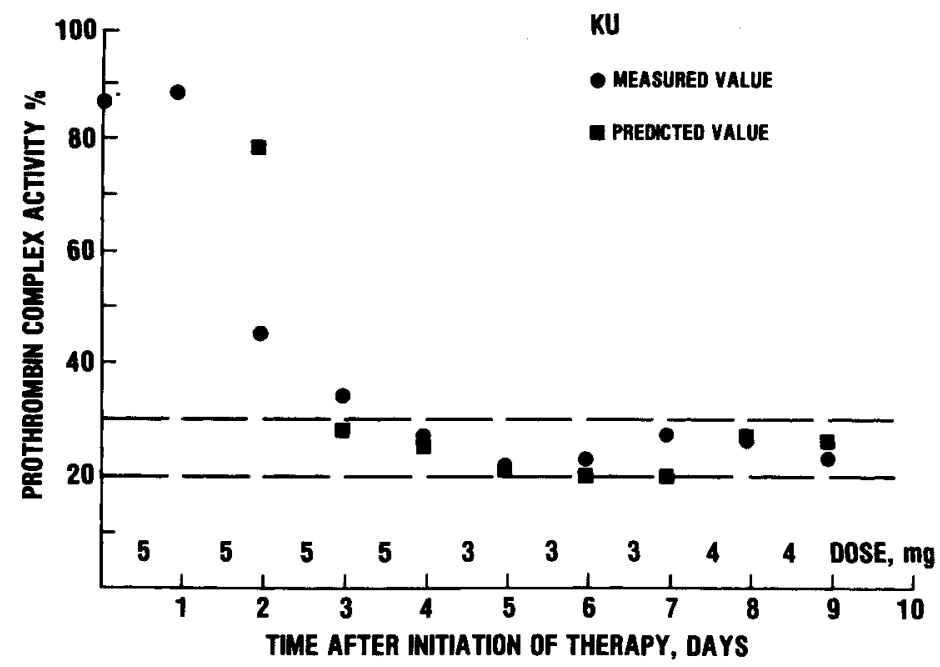

Fig. 3. Measured and predicted PCA responses for patient K. U. (group P-1) in prospective study with program assistance.

assisted group. The agreement between the measured and the predicted PCA values is good and improves as the number of days of therapy increases.

The number of days required to bring the PCA into the therapeutic (20\% to $30 \%$ ) range and the percentage of subsequent days within the therapeutic range were computed for each member of the computer-assisted and control groups (Table III). When distributional requirements appeared to be satisfied, the results were tested for significance by Student's $t$ test. Otherwise, the Wilcoxon rank-sum test was used. The average number of days required to first reach the therapeutic range was greater for the computer-assisted than for the control patients $(P<0.05$ by Student's $t$ test). This additional time, however, did not prolong patients' hospital stays; continued hospitalization was required for other reasons. More importantly, after once having reached the therapeutic range, the program-assisted group remained within the therapeutic range for a much larger percentage of the time $(P<0.01)$. This indicates that the maintenance dose was estimated with much greater accuracy in the computer-assisted group.

It is apparent from Table III that the difference in percentage of time spent within the therapeutic range for the two groups reflects primarily the fact that the program-assisted patients were not overanticoagulated during therapy. This suggests that computer-assisted patients should be at a lower risk of hemorrhage than those who did not receive computerassisted therapy.

\section{Discussion}

We have presented results of what we believe to be the first clinical use of a mathematic model of warfarin dynamics to guide initiation of oral anticoagulant therapy. We did both retrospective and prospective evaluations of a computer program based on the model, which used data obtained both in our laboratory and from the literature. The program does a creditable job of predicting patient response to anticoagulants in the retrospective studies, thus indicating that the model is a reasonable empirical representation of the physiologic system's performance during the initial phases of anticoagulation. These results are confirmed by prospective study. We demonstrated that the use of such a program results in the PCA values remaining within the therapeutic range for an increased percentage of the time.

Rapid identification of a patient's kinetic and dynamic parameters is probably only one reason for the program's increased success in maintaining patients within the therapeutic range. It also 


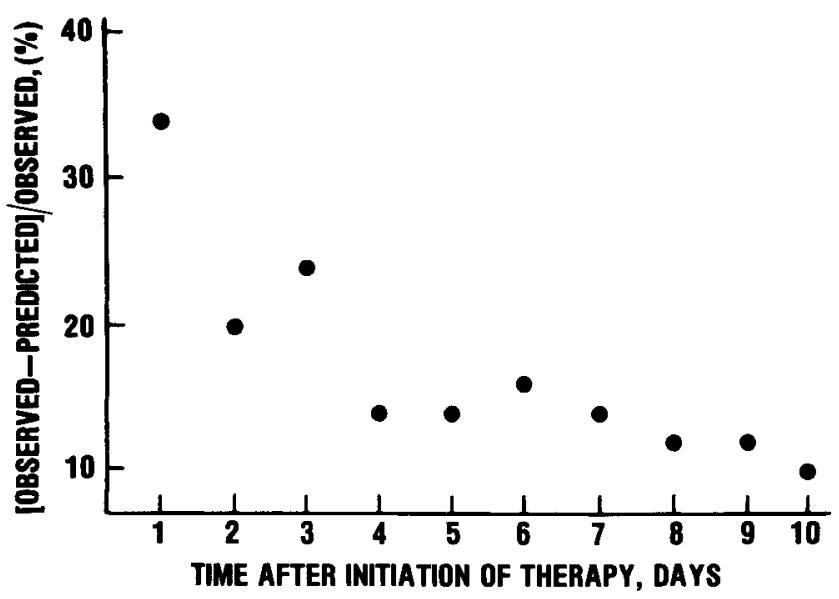

Fig. 4. Average percent deviations of predicted PCA values from observed values for all patients anticoagulated with program assistance (group P-1).

Table III. Prospective comparison of computer-assisted and control groups

\begin{tabular}{|c|c|c|c|}
\hline & $\begin{array}{c}\text { Control } \\
\text { (group } P-C \text { ) }\end{array}$ & $\begin{array}{l}\text { Computer-assisted } \\
\text { (group } P-1)\end{array}$ & $P$ \\
\hline $\begin{array}{l}\text { Percent days within } \\
\text { therapeutic range* }\end{array}$ & $60(33-100)$ & $83(40-100)$ & $<0.01 \dagger$ \\
\hline $\begin{array}{l}\text { Percent days below } \\
\text { therapeutic range* }\end{array}$ & $29(0-50)$ & $6(0-60)$ & $<0.05$ \\
\hline $\begin{array}{l}\text { Percent days above } \\
\text { therapeutic range* }\end{array}$ & $11(0-67)$ & $11(0-50)$ & N.S. $\dagger$ \\
\hline $\begin{array}{l}\text { Days required to achieve } \\
\text { therapeutic range }\end{array}$ & $4.33(1-8)$ & $6.00(4-9)$ & $<0.05 \ddagger$ \\
\hline
\end{tabular}

Data in parenthesis are ranges.

*After first achieving therapeutic range.

$\Varangle$ By Wilcoxon rank-sum test.

$\ddagger$ By Student's t test.

appears likely that the program's maximum dose limitations reduce the probability of giving a dose early in therapy that will drive the PCA below $20 \%$. Once the patient parameters have been identified adequately, the program also tends to limit the frequency and magnitude of changes in dosage in response to small changes in PCA. This may counteract a tendency of inexperienced physicians to react to every change in PCA with a concomitant change in warfarin dose, with resultant overcontrol and undesirable cycling of PCA above and below the desired limits.

Correlation methods, such as those relating ultimate maintenance requirements to the PCA values at a fixed time during a standard loading therapy, seem less satisfactory than the method we have presented. Dosages obtained by the correlation methods may vary widely from the "true" maintenance doses in some patients. Such methods often use only a small portion of the available data from each patient. In contrast, the computer-assisted approach we present here uses all the prothrombin and dosage information obtained on each patient. The method updates its predictions and recommendations as each new datum is obtained and is applicable at any stage of therapy. Finally, it is capable of accounting for changes in the patient's physiologic status with time, as reflected in kinetic and dynamic parameters.

Although further studies are required to de- 
termine the method's range of applicability, results thus far support our belief that the computer-assisted approach described here may lead to improved control of oral anticoagulation.

\section{References}

1. Aggeler PM, O'Reilly RA: Studies on coumarin anticoagulant drugs: initiation of warfarin therapy without a loading dose. Circulation 38: 169-177, 1968.

2. Brotman I: Anticoagulation in myocardial infarction. Am J Cardiol 1:260-270, 1958.

3. Hoffer EP, Marble KM, Yurchak PM, Barnet GO: A computer-based information system for managing patients on long-term oral anticoagulants. Comput Biomed Res 8:573-579, 1975.

4. Jones BR, Baran A, Reidenberg MM: Evaluating patient's warfarin requirements. Clin Res 26:610A, 1978.

5. Morrison GW: Predicting warfarin requirements. Lancet 1:167-168, 1979. (Letter.)

6. Mosely PH, Schatz IJ, Breneman GM, Keyes JW: Long-term anticoagulation therapy. JAMA 186:914-916, 1963.

7. Nagashima R, O'Reilly RA, Levy G: Kinetics of pharmacologic effects in man: the anticoagulant action of warfarin. Clin Pharmacol Ther 10: 22-35, 1968.

8. O'Leary TJ, Abbrecht PH, Powers WF: A computer program for predicting patient response to oral anticoagulants. Clin Res 25:633A, 1977.

9. O'Leary TJ, Abbrecht PH: Predicting oral anticoagulant response using a pharmacodynamic model. Ann Biomed Engr 9:199-216, 1981.

10. Pollard JW, Hamilton MJ, Christensen NA,
Achor RW: Problems associated with long term anticoagulant therapy. Circulation 25:311-317, 1962.

11. Powers WF, Abbrecht PH, Covell D, DuLeep $\mathrm{KH}$ : Optimization applications in anticoagulant therapy. AIAA paper No. 76-804, AIAA/AAS Astrodynamics Conference, San Diego, California, August 1976.

12. Rutledge PA, Davies DM, Bell SM, Cavanagh JS, Rawlins MD: Predicting patients' warfarin requirements. Lancet 2:854-855, 1977.

13. Sawyer WT: Predictability of warfarin dose requirements: theoretical considerations. J Pharm Sci 68:432-434, 1979.

14. Sellers EM, Koch-Wesser J: Kinetics and clinical importance of displacement of warfarin from albumin by acidic drugs. Ann NY Acad Sci 179:213-223, 1971.

15. Shapiro CM, Lister R, Lichtman AM, Josephson AM: Comparative clinical study of coumadin sodium and dicumerol in patients with thromboembolic diseases. Am Heart J 55:66-72, 1958.

16. Sheiner LB: Computer-aided long term anticoagulation therapy. Comput Biomed Res 2:507$518,1969$.

17. Theophanus TB, Barile RG: Multiple-dose kinetics of oral anticoagulants: Methods of analysis and optimized dosing. J Pharm Sci 62:261$266,1973$.

18. Wiegman H, Vossepoel AM: A computer program for long term anticoagulation control. Comput Programs Biomed 7:71-84, 1977.

19. Williams DW, Karl RC: A simple technic for predicting daily maintenance dose of warfarin. Am J Surg 137:572-576, 1979. 